\title{
ADAPTIVE CREDIT SCORING WITH ANALYTIC HIERARCHY PROCESS
}

\author{
Kwang Yong Koh* \\ School of Information Systems \\ Singapore Management University \\ Singapore \\ E-mail: kykoh.2012@mitb.smu.edu.sg \\ Murphy Choy \\ School of Information Systems \\ Singapore Management University \\ Singapore \\ E-mail: murphychoy@smu.edu.sg \\ Michelle L.F. Cheong \\ School of Information Systems \\ Singapore Management University \\ Singapore \\ E-mail: michcheong@smu.edu.sg
}

\begin{abstract}
Credit risk assessment for consumers has been a cornerstone of risk management in financial institutions and constitutes a component of the three pillars of Basel II. Traditionally, the concept of 5 ' $\mathrm{C}$ 's was widely adopted by financial institutions as the key basis for credit risk assessment for loan applications by prospective borrowers. With the evolution of the credit risk management practices, more quantitative methods such as credit scorecards have been developed, which is implemented through the use of logistic regression, decision trees and neural networks. However, such approaches proved to be inadequate with the validity and effectiveness of the approaches doubted especially in the light of the 2008 sub-prime financial crisis in US, which was partly triggered by poor quantitative modeling as well as over-reliance on mathematical modeling resulting in a divorce between reality and model. To remedy the problem of over-reliance on pure quantitative models, Clark Abrahams and Mingyuan Zhang introduced the Comprehensive Credit Assessment Framework (CCAF) that attempts to address weaknesses in the existing credit risk assessment system, and provides flexibility with better accuracy, transparency and simplicity for the various stakeholders in the credit lending business. Unlike the 5 ' $\mathrm{C}$ 's of credit assessment which provides very coarse segregation of potential borrower and focus more on past performance which can be a reflection of better credit environment and no longer represent the current environment, CCAF caters for a more fine-grain segmentation that allow for specific action for specific groups of borrower which results in an adaptive framework that takes in new inputs to improve its predictiveness. This paper proposes to implement the CCAF by utilizing Analytic Hierarchy Process (AHP) for consumer credit segment to establish a new hybrid version of the framework - AHP-CCAF model. The new model is first tested on 3 classical credit risk data to illustrate the feasibility of the model. The paper compares the performance of the new model against traditional method of Decision Tree Analysis. Results shows that the proposed model is feasible and has better forecasting capability than the traditional methods.
\end{abstract}

\footnotetext{
${ }^{*}$ Corresponding author
} 
Keywords: Credit Risk, Credit Score Card, Decision Tree, Comprehensive Credit Assessment Framework (CCAF), Analytic Hierarchy Process (AHP), Basel II.

\section{Introduction}

Traditionally, the concept of 5 'C's was widely adopted by financial institutions as the key basis for credit risk assessment for loan applications by prospective borrowers. Over the years, credit risk management practices had evolved, and more quantitative methods such as credit scorecards have been developed.

Such approaches were proved to be inadequate, especially in during the 2008 sub-prime financial crisis, which was assessed to be partly triggered by poor quantitative modeling as well as over-reliance of mathematical modeling. To remedy the problem on over-reliance on pure quantitative models, Clark Abrahams and Mingyuan Zhang introduced the Comprehensive Credit Assessment Framework (CCAF) (Clark Abrahams, 2009) that attempted to address weaknesses in the existing credit risk assessment system, and proposed to provide flexibility with better accuracy, transparency and simplicity for the various stakeholders in the credit lending business. CCAF caters for a more fine-grain segmentation that allow for specific action for specific group of borrower, and it provides an adaptive framework to take in new inputs to improve its predictiveness. This paper proposes to implement the CCAF by utilizing Analytic Hierarch Process (AHP) for consumer credit segment by establishing a new hybrid version of the framework.

\section{Background}

Three key framework and techniques were used in this paper. This section provides some background on the individual methods and discusses the individual method's relevance to the new proposed CCAF/AHP framework.

\section{Comprehensive Credit Assessment Framework}

The Comprehensive Credit Assessment Framework (CCAF) by Clark Abrahams and Mingyuan Zhang provided the framework to derive different group of borrowers (termed as handlers). This was achieved by providing a sustainable and sensible segmentation based on the primary credit factors. The model consensus session (MCS) is a core mechanism with the framework, where "CCAF ensures that classification of credit transactions is performed sensibly and comprehensively."

The segmentation would results in two key profiles - termed as Borrower's Contour (BC) and Transaction Contour (TC). In Borrower's Contour, CCAF classifies borrowers by a selected set of attributes. The core group of attributes used to classify the Borrower's Contour is considered as the primary factors. The composition of the primary factors would result in the corresponding hard handlers used to segment the different types of borrowers. With the established BC, the corresponding Transaction Contour would be derived to determine the likelihood of default for a particular hard handler type. In the CCAF framework, expert judgment was used to determine the likelihood of default for a particular type of borrower segment. In this paper, the hybrid model proposes to use the historical data to determine the likelihood of default as the basis of classification.

\section{Analytic Hierarchy Process}

The Analytic Hierarchy Process (AHP) method (Saaty, 1980) by Thomas L. Saaty provided the mathematical rigorousness for decision making that involves multiple criteria. The AHP method was an approach to break down a complex problem (involving multiple considerations or attributes) and to combine the solutions to the subproblems into an overall conclusion. Furthermore, by providing a

\footnotetext{
${ }^{1}$ (Clark Abrahams, 2009) Pg 49
} 
relative scale of 1-9 (Saaty, Decision Making with the Analytic Hierarchy Process, 1998) to rank importance between two variables or attributes, it provided a systematic way for pairwise comparison of both tangible and intangible factors. For $n$ number of attributes, it would involve $n(n-1) / 2$ pairwise comparisons.

A typical AHP analysis could be represented by a matrix of pairwise ratios, where rows give the ratios of the weightage or relative importance of each attribute with respect to all others. Consider $\mathrm{n}$ attributes, A1, ..,An with known worth w1, .., wn respectively (Saaty T. L., Relative Measurement and Its Generalization in Decision Making. Why Pairwise Comparisons are Central in Mathematics for the Measurement of Intangible Factors.The Analytic Hierarchy/Network Process, 2008):

$$
\begin{aligned}
& \text { Al } \cdots \quad A n
\end{aligned}
$$

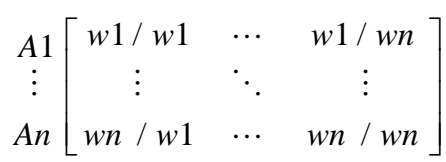

where the scale $\mathrm{w}$ can be recovered using the following equation:

$$
\left[\begin{array}{ccc}
w 1 / w 1 & \cdots & w 1 / w n \\
\vdots & \ddots & \vdots \\
w n / w 1 & \cdots & w n / w n
\end{array}\right]\left[\begin{array}{c}
w 1 \\
\vdots \\
w n
\end{array}\right]=n\left[\begin{array}{c}
w 1 \\
\vdots \\
w n
\end{array}\right]
$$

or briefly $\mathrm{Aw}=\mathrm{nw}$. To make $\mathrm{w}$ unique, one can normalize the pairwise comparison matrix by dividing each element by its column sum, and set wi equal to the average of the elements in row $\mathrm{i}$ in the normalized matrix.

The rigorousness of mathematical basis using the eigenvector method (T.L. Saaty, 1998) to represent relative ranking between factors ensured consistency of judgment when pairwise comparison was conducted between the factors by tolerating an "error in measurement only when it is of a lower order of magnitude (10 percent) than the actual measurement itself"'. To check for consistency, one can carry out (Anderson, 2002) a matrix multiplication of the pairwise comparison matrix with the column vector of weights, and then compute the sum of the element in this column vector divided by the corresponding element in the weight vector and set $\lambda$ max equal to this sum divided by $n$, the number of attributes. With $\lambda \max$, the consistency index (C.I.) could be computed which is given by:

$$
\text { C.I. }=(\lambda \max -n) /(n-1)
$$

The consistency ratio (C.R.) could then be computed by having consistency index C.I. divided by R.I., where R.I. is defined to be the consistency index of a randomly generated pairwise comparison matrix.

The AHP method has been widely adopted in many different applications (Omkarprasad S. Vaidya, 2006). A related work on application of AHP method was for bankruptcy prediction (Cheol-Soo Park, 2002), but this involved mainly the application of k-nearest neighbor (k-NN) method with the AHP method. For this paper, the AHP method provides a framework to arrange factors in a hierarchic or network structure, and measurements were made by providing a systematic relative scale to rank the different attributes for predicting the likelihood of incurring a bad loan. The pairwise comparison framework provided by the AHP method was used to check for consistency of hard handlers derived from the CCAF framework. It provided a framework to check for consistency for two key scenarios - when the hard handlers were ranked according to the bad loan rate, and when human expert judgment is exercised on the original hard handlers ranking.

\footnotetext{
2 (Saaty, 1980) Pg 84
} 
Proceedings of the International Symposium on the Analytic Hierarchy Process 2013 


\section{Principal Component Analysis}

The Principal Component Analysis (PCA) (Pearson, 1901) by Karl Pearson is a mathematical procedure that allows transformation of a set of observations into a set of values that are linearly uncorrelated variables, termed as principal components. These principal components provided "a reduction of the data without much loss of information" 3 from the original set of data, and were linear combinations that represented independent or orthogonal amounts of variance in a given set of data.

PCA has been commonly used as a form of pre-processing to choose the highest-variance principal components when data sets involved multiple dimensions. Applications include PCA of large (PET) Data sets (K.J. Friston, 1993), handwritten zip code classification (Hastie, 2001) and gene expression data analysis (Misra, 2002). With this method, it provided the mathematical basis to reduce the large number of attributes to a few key attributes (based on the loading of the different attributes on the derived components). The derived key attributes were then used to construct the hard handlers used for CCAF framework.

\section{Method used for CCAF/AHP framework}

This section describes the steps involved in the proposed CCAF/AHP framework.

Data format of a selected set of data was examined to check data type (whether numerical or categorical) and to check for missing data. Categorical data that has more than 7 levels were not selected for analysis as it was assessed to provide too fine-grained breakdown and there was no fixed standard to compare. Numerical data were translated to 5 sets of similar proportions. Similar proportions method was used to prevent extreme outliers that may result in having a particular set with abnormally low count. Observations with missing data were noted, but was included as part of the analysis.

The CCAF framework was used to rank individual selected attribute according to the target rate. In this paper, the bad loan rate is ranked from the lowest to the highest. With the set of selected attributes, the PCA method was used to identify the top 5 attributes that could represent the Borrower Contour in the CCAF framework. Top 5 attributes were selected as the CCAF framework examines the Five Cs of Credit "in context with one another", , so that it provided a more comprehensive classification of the borrower.

The number of levels for each attribute was used to derive the number of permutations expected when $n$ number of attributes was selected to create the Borrower Contour (termed as hard handler). The number of expected permutations was then used for comparison with the number of observations for a selected data set. If the number of observations divided by number of expected permutations was less than 25 , the number of attributes used to create the hard handlers would be reduced by one and the comparison would be repeated. Reason for choosing ratio of 25 as the number was to prevent number of observations to be too small for a particular hard handler. In fact, there might be outliers in the data set, resulting in a skewed distribution of having high numbers of observations for a particular hard handler, and very low number of observations for some other hard handlers. The ratio of 25 was chosen as a guide.

By applying the results of PCA method to the CCAF framework, the derived hard handlers were listed in order according to the corresponding target rate (in this case, bad loan rate was chosen). A plot of hard handlers with corresponding bad loan rate was plotted to observe the relationship. The plot might not be increasing or decreasing monotonically, as was expected in a set of observations from real data. To

\footnotetext{
3 (Rao, 1964) Pg 329

4 (Clark Abrahams, 2009) Pg 52 - "How CCAF Classifies Borrowers"
} 
determine whether the variations in bad loan rate for the different hard handlers (increasing or decreasing in an unexpected manner compared to a monotonous manner), the consistency check from the AHP method was used to check for reasonableness in consistency

Applying consistency check from the AHP method for pairwise comparison of hard handlers provided the mathematical basis to examine whether bad loan rates for the hard handlers were consistent. First, the hard handlers were checked for pairwise comparison in sets of 9 hard handlers. Sets of 9 hard handlers were originally chosen for ease of ranking. This was not limited to 9 hard handlers for comparison, as long as the random index (R.I.) for number of variables above 9 was available. Having worked out the comparison matrix for a selected set of 9 hard handlers, the corresponding normalized matrix was derived. The $\lambda \max$, consistency index (C.I.) and consistency ratio (C.R.) ${ }^{5}$ were derived to check for consistency. If the C.R. was above 0.1 , it was considered as not reasonably consistent, and would require a relook at the choice of attributes used to create the hard handlers.

Decision Tree Analysis was applied to the same set of data, as this was one of the key traditional methods used for bad loan rate prediction (Quinlan, 1987) (Quinlan, C4.5: Programs for Machine Learning, 1993). The corresponding confusion matrix was compared to the results derived from the CCAF/AHP framework. The following parameters were used to calculate the confusion matrix from the CCAF/AHP framework:

a. Average bad loan rate for the entire set of observation was used to predict whether a particular hard handler was likely to result in bad loan or not.

b. For each set of hard handler, the actual bad loan and good loan was used to calculate the corresponding prediction for good and bad loans.

c. With this worked out for each handler set, the total number of actual bad loans (with predict bad loan and predict good loan) and actual good loans (with predict bad loan and predict good loan) could be worked out.

This provided the calculation basis to derive a confusion matrix to compare that with the result from the Decision Tree Analysis. The key comparison parameters between the Decision Tree Analysis and CCAF/AHP framework were:

a. Overall misclassification rate

b. Correct Prediction rate

c. False Negative Rate - defined as Actual Good loan predicted as Bad Loan, divided by Total number of Good Loan. This is relevant for the case of CCAF framework as it aims to be better than conventional credit scoring models that "typically lump chronic late payers who pay late fees...together with actual defaulters on obligations, which overstates the risk and results in the consumer being overcharged for credit"

\section{Data used to test the CCAF/AHP framework}

Three set of data were used for the analysis of CCAF/AHP framework. The first set was a data set of 1000 observations for German credit data, provided by Professor Dr. Hans Hofmann from Institut für Statistik und Ökonometrie Universität Hamburg. This set of data contained 24 attributes - 18 categorical data and 6 numerical data, with one target attribute to represent good or bad debt. There were no missing data for all 24 attributes in these 1000 observations.

\footnotetext{
5 (Saaty, 1980) Pg 84

6 (Clark Abrahams, 2009) Pg 53
} 
The second data set were 690 observations for Australian credit approval data, provided by Ross Quinlan from his paper on "Simplifying decision trees" (Quinlan, Simplifying Decision Trees, 1987). This set of data contained 14 attributes -8 categorical data and 6 numerical data, with one target attribute to represent good or bad debt. There were 37 cases of observations with one or missing values for the 14 attributes, and were replaced by the mode of the attribute for categorical data, and mean of the attribute for numerical data. The replacement of the missing data was handled by the original data set.

The third data set were 690 observations for Japanese credit screening, provided by Chiharu Sano from Donald Bren School of Information and Computer Sciences at University of California. This set of data contained 15 attributes - 9 categorical data and 6 numerical data, with one target attribute to represent good or bad debt. Missing data were applied with the same consistent value for the different attributes and were used as part of the analysis.

\section{Results}

The results from these three sets of data are shown, first using the CCAF/AHP framework, followed by the Decision Tree Analysis, and the comparison between these two methods.

\section{A. Results for German Data using CCAF/AHP Framework}

PCA method was applied to select 5 out of 24 possible attributes. Using the guideline of having at least 25 observations per handler, the first 4 attributes are chosen to derive the hard handlers. The chosen first 4 attributes (terms as set of 4 hard handlers) were used to create 40 hard handlers, and it was observed that the bad loan rate was in a haphazard manner. However, a pattern was observed that when treating the $4^{\text {th }}$ attribute (with 2 levels) as a potential soft handler (i.e. using a $3+1$ set of handlers), there was an increasing pattern for both cases when the $4^{\text {th }}$ attribute is level 1 (Figure 1).

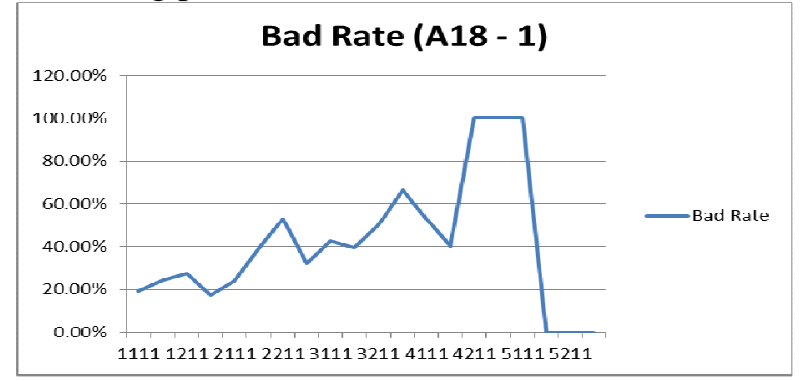

Figure 1 Three Hard Handlers with One Soft Handler (A18) with level 1 for German Data

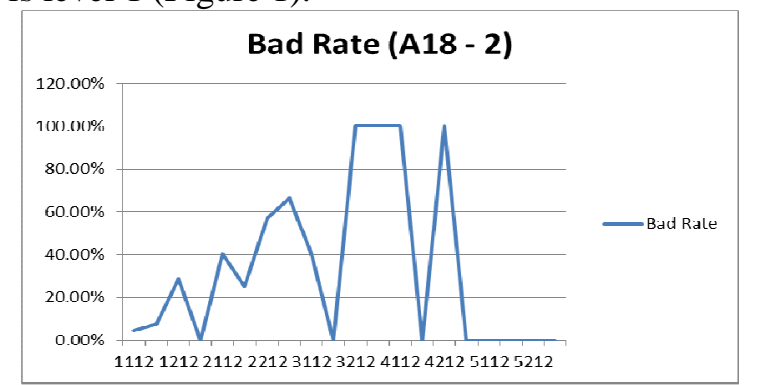

Figure 2 Three Hard Handlers with One Soft Handler (A18) with level 2 for German Data

Figure 2 shows that when soft handler (A18) has a value of 2 , the graph was not in an expected manner of general upward trend. An attempt was next made to reduce the handlers to 3 hard handlers (termed as 3 hard handlers). The corresponding plot of 3 hard handlers was plotted in Figure 3.

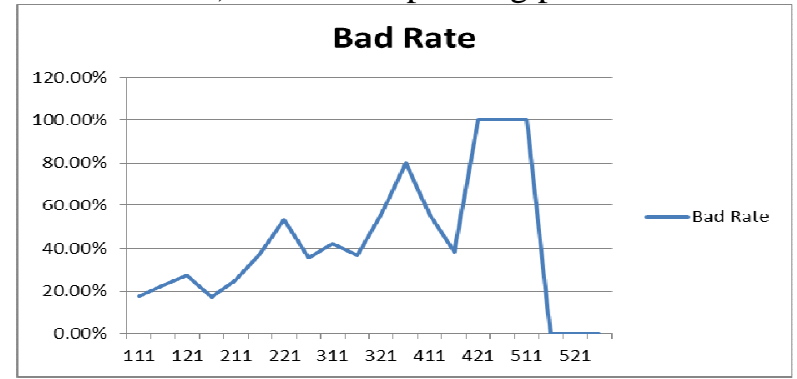

Figure 3 Three Hard Handlers for German Data

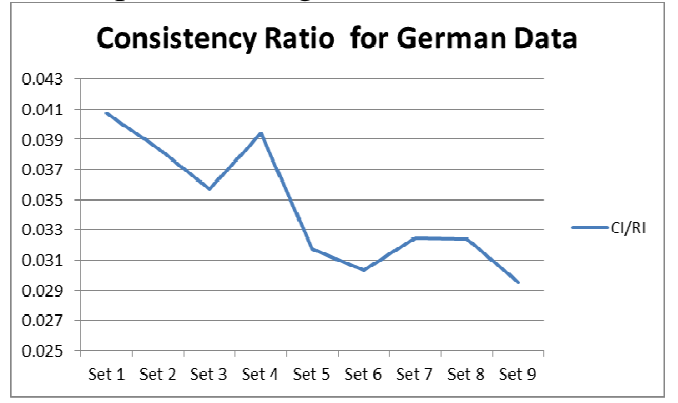

Figure 4 Plot of C.R. for German Data 
Figure 3 (with three hard handlers) was shown to exhibit similar pattern to that of Figure 2 (with three hard handlers and one soft handler with value of 1). Since the three hard handlers showed to have similar profile, these three hard handlers were chosen for subsequent analysis.

The chosen three hard handlers have a permutation of 20 handlers. Amongst these 20 possible handlers, 3 handlers did not have any observations, and hence were not used in the pairwise comparison. With the remaining 17 handlers and using set of 9 handlers for pairwise comparison, 9 sets of such comparisons were derived and check for consistency. The C.R. for these 9 sets of comparisons is shown in Figure 4, with average C.R. calculated as 0.0345 and standard deviation of 0.0041. Using the AHP framework for consistency check showed that the chosen set of 17 hard handlers are reasonably consistent with increasing bad loan rate for the German data.

\section{B. Results for Australia Data using CCAF/AHP Framework}

PCA method was then applied to select 5 out of 12 possible attributes. The first 2 attributes are chosen to create 25 hard handlers. Figure 5 shows a general increasing trend for the higher levels of handlers.

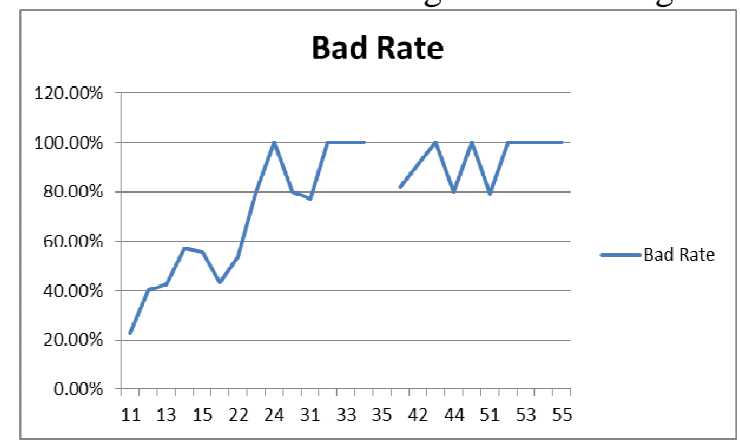

Figure 5 Two Hard Handlers for Australia Data

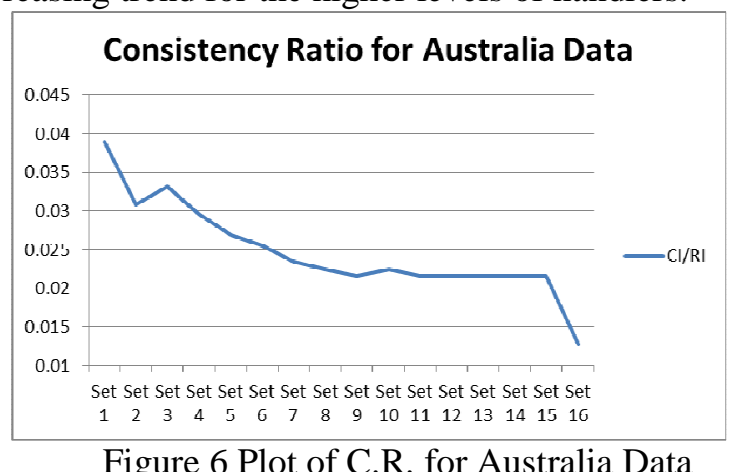

Figure 6 Plot of C.R. for Australia Data

The chosen two hard handlers have a permutation of 25 handlers. Amongst these 25 possible handlers, 1 handler did not have any observations, and hence were not used in the pairwise comparison. With the remaining 24 handlers and using set of 9 handlers for pairwise comparison, 16 sets of such comparisons were derived and check for consistency. The C.R. for these 16 sets of comparisons is shown in Figure 6, with average C.R. calculated as 0.0247 and standard deviation of 0.0061. Using the AHP framework for consistency check showed that the chosen set of 24 hard handlers are reasonably consistent with increasing bad loan rate for the Australia data.

\section{C. Results for Japan Data using CCAF/AHP Framework}

PCA method was then applied to select 5 out of 13 possible attributes. The chosen first 2 attributes were used to create 15 hard handlers. Figure 7 shows a general increasing trend for the higher levels of handlers, with gaps in the gap representing handlers with no observations.

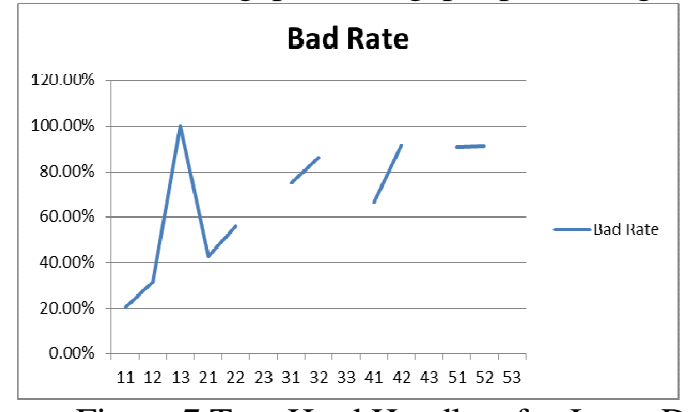

Figure 7 Two Hard Handlers for Japan Data

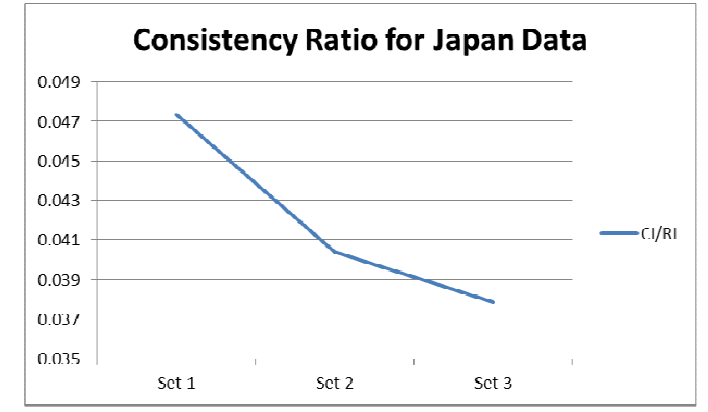

Figure 8 Plot of C.R. for Japan Data 
The chosen two hard handlers have a permutation of 15 handlers. Amongst these 15 possible handlers, 4 handlers did not have any observations, and hence were not used in the pairwise comparison. With the remaining 11 handlers and using set of 9 handlers for pairwise comparison, 3 sets of such comparisons were derived and check for consistency. The C.R. for these 3 sets of comparisons is shown in Figure 8, with average C.R. calculated as 0.0419 and standard deviation of 0.0049. Using the AHP framework for consistency check showed that the chosen set of 11 hard handlers are reasonably consistent with increasing bad loan rate for the Japan data.

\section{D. Results for German Data using Decision Tree Analysis and Comparison with CCAF/AHP method}

The same set of German Data was analysed using Decision Tree Analysis. The 1000 observations from German Data were randomly split into $80 \%$ for Training, and $20 \%$ for Validation. The average bad loan rate for German Data was 30\%, and this was used as the deciding factor to predict whether a particular handler is likely to result in bad loan or not for the CCAF/AHP method. Figure 9 shows the overall comparison between the unpruned Decision Tree, pruned Decision Tree and the CCAF/AHP method. The red cells indicate areas that CCAF/AHP method performed poorer than the Decision Tree analysis, and green cells indicate areas that CCAF/AHP method performed better. In this case, it showed that the AHP method was able to provide prediction decision ability at close to $100 \%$ power with a reduction in false negative rate, when compared to the pruned Decision Tree method.

\begin{tabular}{|c|c|c|c|c|c|}
\hline \multicolumn{6}{|l|}{ German Data Set } \\
\hline & Decision Tree (Unpruned) & Decision Tree (Pruned) & AHP Method & vs Unpruned & vs Pruned \\
\hline & Split $14 \quad 9$ variables & Split $3 \quad 3$ variables & 3 variables & & \\
\hline overall misclassification & $26.86 \%$ & $33.14 \%$ & $34.00 \%$ & $7.14 \%$ & $0.86 \%$ \\
\hline Predicts correctly & $73.14 \%$ & $66.86 \%$ & $66.00 \%$ & $-7.14 \%$ & $-0.86 \%$ \\
\hline False Negative Rate* & $16.10 \%$ & $28.81 \%$ & $28.14 \%$ & $12.04 \%$ & $-0.67 \%$ \\
\hline
\end{tabular}

Figure 9 Comparison of three methods for German Data

\section{E. Results for Australia Data using Decision Tree Analysis and Comparison with CCAF/AHP method}

Similarly, the 690 observations from the Australia Data was randomly split into 80\% for Training, and $20 \%$ for Validation, and was analysed using Decision Tree Analysis. The average bad loan rate for Australia Data was $44.5 \%$, and this was used as the criteria to predict whether a particular handler will incur bad loan or not for the CCAF/AHP method. Figure 10 shows the comparison between the methods for Australia data. It showed that the AHP method was able to provide prediction decision ability at $90 \%$ of the predictive power of Decision Tree method, with a reduction in false negative rate.

\begin{tabular}{|c|c|c|c|c|c|}
\hline \multirow[t]{2}{*}{ Australia Data Set } & & \multirow[b]{2}{*}{ Decision Tree (Pruned) } & \multirow[b]{2}{*}{$\begin{array}{l}\text { AHP Method } \\
2 \text { variable }\end{array}$} & \multirow[b]{2}{*}{ vs Unpruned } & \multirow[b]{2}{*}{ vs Pruned } \\
\hline & Decision Tree (Unpruned) & & & & \\
\hline overall misclassification & $15.13 \%$ & $15.97 \%$ & $25.07 \%$ & $9.95 \%$ & $9.11 \%$ \\
\hline Predicts correctly & $84.87 \%$ & $84.03 \%$ & $74.93 \%$ & $-9.95 \%$ & $-9.11 \%$ \\
\hline False Negative Rate* & $23.29 \%$ & $23.29 \%$ & $9.92 \%$ & $-13.37 \%$ & $-13.37 \%$ \\
\hline
\end{tabular}

Figure 10 Comparison of 3 methods for Australia Data

5F. Results for Japan Data using Decision Tree Analysis and Comparison with CCAF/AHP method The 690 observations for Japan Data were also randomly split into $80 \%$ for Training, and 20\% for Validation. This data set was analysed using Decision Tree Analysis. The average bad loan rate for Japan Data was $44.5 \%$, and this rate was used as the decision point to predict whether a handler will result in bad loan or not for the CCAF/AHP method. Figure 11 shows the comparison between the three 
method. Similar to the Australia data, it showed that the AHP method was able to provide prediction decision ability at $90 \%$ power with a reduction in false negative rate, when compared to the pruned Decision Tree method.

\begin{tabular}{|c|c|c|c|c|c|}
\hline \multirow[t]{3}{*}{ Japanese Data Set } & & & \multirow{3}{*}{$\begin{array}{l}\text { AHP Method } \\
2 \text { variables }\end{array}$} & \multirow{3}{*}{ vs Unpruned } & \\
\hline & Decision Tree (Unpruned) & Decision Tree (Pruned) & & & \multirow[t]{2}{*}{ vs Pruned } \\
\hline & Split $8 \quad 7$ variables & Split $2 \quad 2$ variables & & & \\
\hline overall misclassification & $15.13 \%$ & $14.47 \%$ & $25.94 \%$ & $10.81 \%$ & $11.47 \%$ \\
\hline Predicts correctly & $84.87 \%$ & $85.53 \%$ & $74.06 \%$ & $-10.81 \%$ & $-11.47 \%$ \\
\hline False Negative Rate* & $2.38 \%$ & $20.24 \%$ & $10.97 \%$ & $8.59 \%$ & $-9.27 \%$ \\
\hline
\end{tabular}

Figure 11 Comparison of 3 methods for Japan Data

\section{Discussion and Interpretation of the Results}

Key findings from the results were:

a. For all three data sets, the CCAF/AHP framework was able to achieve acceptable consistency ratio for the hard handlers derived and used for differentiating between the different types of loan types.

b. This showed that CCAF/AHP framework was able to consistently provide a basis of ranking between the different handlers, and would be a useful tool for deciding whether to grant a loan or not

c. For Australia and Japan Data, the CCAF/AHP framework was able to provide prediction decision ability at $90 \%$ of the prediction power of decision tree, with a reduction in false negative rate, when compared to pruned decision tree performance from the decision tree analysis.

d. For German Data, the CCAF/AHP framework was able to even perform better as its prediction decision ability was almost on par with the pruned decision tree performance.

e. This showed that the CCAF/AHP framework was a practical and viable framework to be applied to decide whether loans should be granted to a particular handler (or known as a particular profile of loan applicant).

\section{Further Work and Flexibility of the CCAF/AHP framework}

By leveraging on the consistency check and consistency ratio of the AHP method, the CCAF/AHP framework provides the platform to allow inclusion of expert judgment and to cater for inclusion of soft handlers to better classify the borrowers. Adjustments could be made to each pairwise comparison of attributes, while at the same time, the consistency ratio could be kept in check for reference.

This makes the CCAF/AHP framework a flexible framework that could cater for expert judgment and yet at the same time, adhere to the consistency check of the AHP method.

\section{Conclusion}

In conclusion, this paper extends the CCAF framework by incorporating the AHP method to check for pairwise comparison of the attributes and to ensure consistency during the pairwise comparison. PCA analysis was used to reduce the number of dimensions to determine the more representative attributes as inputs to the CCAF/AHP framework analysis. Three sets of data - German, Australia and Japan Data, were in turn applied to the proposed framework and check for consistency of the proposed ranking from the derived set of hard handlers. All three sets of data showed that the CCAF/AHP framework was able to provide consistency to the pairwise comparison of the hard handlers. 
To illustrate the performance of the proposed framework, it was compared against the conventional Decision Tree Analysis. Two key comparisons were made - one versus the unpruned Decision Tree analysis, and another was versus the pruned Decision Tree analysis (corresponding to the number of attributes used to derive hard handlers). Results showed that for the Australia and Japan Data, the CCAF/AHP framework was able to provide prediction decision ability at $90 \%$ power with a reduction in false negative rate, when compared to the corresponding pruned decision tree analysis. The proposed framework was able to perform even better when the German data was used for comparison. Further work to extend the flexibility of the CCAF/AHP framework was discussed, as it allowed incorporation of expert judgment and yet at the same time provided a sound framework to ensure consistency of the changes made.

In summary, the proposed CCAF/AHP framework was a useful method to propose a set of hard handlers for classification and ranking of a particular performance parameter. The performance of this framework was shown to be consistent using three sets of data for comparison and further work could involve increasing the flexibility of the framework by allowing expert judgment.

\section{REFERENCES}

Anderson, D. D. (2002). An Introduction to Management Science: Quantitative Approaches to Decision Making. Mason, Ohio: South-Western Publishing.

Cheol-Soo Park, I. H. (2002). A case-based reasoning with the feature weights derived by analytic hierarchy process for bankruptcy prediction. Expert Systems with Applications.

Clark Abrahams, M. Z. (2009). Credit Risk Assessment - The New Lending System for Borrowers, Lenders and Investors. New Jersey: John Wiley \& Sons, Inc.

Hastie, T. T. (2001). The Elements of Statistical Learning; Data mining, Inference and Prediction. New York: Springer Verlag.

K.J. Friston, C. F. (1993). Functional Connectivity: The Principal-Component Analysis of Large (PET) Data Sets. Journal of Cerebral Blood Flow and Metabolism, 5-14.

Misra, J. S. (2002). Interactive exploration of microarray gene expression patterns in a reduced space. Genome Research 12, 1112-1120.

Omkarprasad S. Vaidya, S. K. (2006). Analytic hierarchy process: An overview of applications. European Journal of Operational Research, 1-29.

Pearson, K. (1901). On Lines and Planes of Closest Fit to Systems of Points in Space. London.

Quinlan, J. R. (1987). Simplifying Decision Trees. International Journal of Man-Machine Studies 27(3), $221-234$.

Quinlan, J. R. (1993). C4.5: Programs for Machine Learning. Morgan Kaufmann.

Rao, C. R. (1964). The Use and Interpretation of Principal Component Analysis in Applied Research. Sankhyā: The Indian Journal of Statistics, Series A (1961-2002), 329-358.

Saaty, T. L. (1977). A scaling method for priorities in hierarchical structures. Journal of Mathematical Psychology, Vol. 15, No. 3, 234-281.

Saaty, T. L. (1980). How to Make A Decision: The Analytic Hierarchy Process. In The Analytic Hierarchy Process (pp. 75-105). New York: McGraw Hill.

Saaty, T. L. (1998). Decision Making with the Analytic Hierarchy Process. Int. J. Services Sciences, Vol.1, No.1, 8398.

Saaty, T. L. (1998). Ranking by Eigenvector Versus Other Methods in the Analytic Hierarchy Process. Appl. Math. Lett Vol. 11, No. 4, 125-128.

Saaty, T. L. (2008). Relative Measurement and Its Generalization in Decision Making. RACSAM, 251-318. 